\title{
Scattering of Low-Energetic Atoms and Molecules from a Boron-doped CVD Diamond Surface
}

\author{
M. Allenbach ${ }^{*}$, M.B. Neuland', A. Riedo', and P. Wurz \\ Unjversity of Bern, Physics 1nstitute, Space Research and Planetary Sciences, Sidlerstrasse 5, 3012 \\ Bern, Switzerland
}

Keywords: Low energetic atom imaging, Chemical vapor deposition diamond, Charge state conversion surface, Ion scattering. Laser ablation ionization mass spectrometer, Space research

No color for any figures in print

\section{Highlights}

1) B-doped synthetic diamond surface successfully tested for space application.

2) Angular scatter of low-energy $\mathrm{H}$ and $\mathrm{O}$ ions is less than that of other synthetic diamond samples.

3) Boron-doping allowed preventing electrostatic charging of synthetic diamond material.

\begin{abstract}
For the detection of low energetic neutral atoms for the remote sensing of space plasmas, charge state conversion surfaces are used to ionize the neutrals for their subsequent measurement. We investigated a boron-doped Chemical Vapo: Deposition (CVD) diamond sample for its suitability to serve as a conversion surface on future space missions, such as NASA's Interstellar Mapping and Acceleration Probe.

For $\mathrm{H}$ and $\mathrm{O}$ atoms incident on conversion surface with energies ranging from 195 to $1000 \mathrm{eV}$ and impact angles from $6^{\circ}$ to $15^{\circ}$ we measured the angular scattering distributions and the ionization yields. Atonic force microscope and laser ablation ionization mass spectronetry analyses were applied to further characterize the sample. Based on a figure-of-merit, which included the ionization yield and angular scatter distribution, the B-doped CVD surface was compared to other, previously

" Corresponding Author: allenbachmarc@hotmait.com (M. Allenbach)

I maike.neuland@irf.se, present address: Swedish Institute of Space Physics (JRF), Rymdcampus 1, 98128

Kiruma, Sweden

? riedo@strw.leidemuniv.nl, present address: Sackler Laboratory for Astroplysics, Leiden Observatory, Lciden

University, Niels Bohrweg 2, 2333 CA Leiden, The Nederlands

${ }^{3}$ peter.wirz@space.unibe.ch
\end{abstract}


characterized conversion surfaces, including e.g. an undoped CVD diamond with a metallized backside. For particle energies below $390 \mathrm{eV}$ the performance of the B-doped CVD conversion surfaces is comparable to surfaces studied before. For higher energies the figure-of-merit indicates a superior performance. From our studies we conclude that the B-doped CVD diamond sample is well suited for its application on future space missions.

\section{Introduction}

The measurement of Energetic Neutral Atoms (ENAs) is a powerful technique to study space plasma distributions via remote sensing. whereby plasma ions charge exchange with the neutral ambient gas [1]. The newly created ENAs keep the velocity and the direction of the original plasma ions but since they are not bound by the electromagnetic forces anymore, they travel along ballistic trajectories. This allows for remote observation of space plasmas. Requirements for ENA instrumentation are the registration of the ENA, its mass and energy, and its direction to obtain the full information on the plasma distribution. A crucial step is the ionization of the ENA. For ENAs with energies of only a few hundreds of $\mathrm{eV} / \mathrm{q}$, the only possibility for the ionization is the application of a conversion surface [1]. The ENAs are scattered from a suitable conversion surface, where a considerable fraction of the scattered particles are either positive or negative ions [1]. ENA imaging instruments for positive as well as for negative ions have been realized, for example [2,3]. Examples of ENA inaging by these instruments are presented in [4], for positive ions and in [5], for negative ions.

A key component in the ENA imaging instrument is the conversion surface. The desired properties of a conversion surface are a ligh ion yield, as well as a narrow angular scattering distribution of the particles from the surface. A high negative ionization yield increases the sensitivity of the instrument and a narrow angular scattering distribution increases the transmission through the ion-optical system [24]. In addition, chemical stability of the surface is desirable. Further the availability of high quality strfaces, at a reasonable price, plays an important role, because depending on the instrument design it can be necessary to cover a large area with conversion surfaces. Dianond was identified already by previous research as a potential material for conversion surfaces, where for polished natural diamond a high ionization yield and good angular scattering properties were presented in [6]. However, the high costs of natural diamond were prohibitive to equip instruments for ENA detection with conversion surfaces of this material. As an alternative, synthetic diamond surfaces, e.g. coatings of free-standing chemical vapor deposited (CVD) diamond were investigated [7-9]. For NASA's Interstellar Boundary Explorer Mission (IBEX) diamond-like conversion surfaces were used in the IBEX-Lo ENA camera [3]. Despite the very successful application of synthetic diamond surfaces in the IBEX mission, the research on diamond based conversion surfaces continues, e.g. [10], looking for improved ionization yields, narrower angular scattering distributions, and easy availability of high quality surfaces. For example, NASA's Intersteliar Mapping Probe (IMAP), the follow up mission of IBEX, aims at measuring interstellar neutral atoms at the boundary of the heliosphere with larger spatial resolution, larger energy range and higher sensitivity than JBEX did [11]. The Jupiter Neutral Atoms Analyser (JNA) onboard ESA's Jupiter Icy Moon Explorer (JUICE) will map neutral atoms to study the Jovian plasma environment and its interaction with the icy surfaces of the Jovian moons [12]. Both, IMAP and JNA require high quality conversion surfaces, the first one especially regarding angular scattering properties, the latter especially regarding chemical inertness and radiation hardness as the conversion surfaces are exposed to strong radiation. With advancing space research, also the requirements on conversion surfaces will increase. Therefore, the constant research on newly available materials and their characterization as potential conversion surfaces is important and of high interest for other space instrumentation applying similar surfaces, for example time-of-flight (TOF) instruments making use of secondary electron emission properties. 


\section{Experiment}

\subsection{Experimental set up ILENA}

Figure 1 shows the schematic of the measurement set up of the Imager for Low Energetic Neutral Atoms (ILENA) test facility at the University of Bern, Switzerland. As the setup has been described in detail in earlier publications $[6,7,13,14]$, only a short description will be given here. An ion beam of the species of interest is produced by an electron-impact ion source. The ions are accelerated to the desired energy and focused by suitable ion optics toward the sector magnet, which deflects the ion beam by $90^{\circ}$. By applying a suitable magnetic field, it is ensured that only the selected atom species reaches the charge state conversion surface (CS). Passing through a pinhole of $1 \mathrm{~mm}$ diameter and further ion optics, the ions are directed onto the test conversion surface, where they are scattered. The sample is mounted on a holder that can be rotated to allow for different angles of incidence. After interaction and charge exchange processes with the surface, a retarding potential analyzer (RPA) prevents positive ions from entering the detection unit and similarly a grid to repel electrons. For position sensitive detection of the negatively charged ions and the neutral atoms, three microchannel plates in Z-Stack configuration are used.

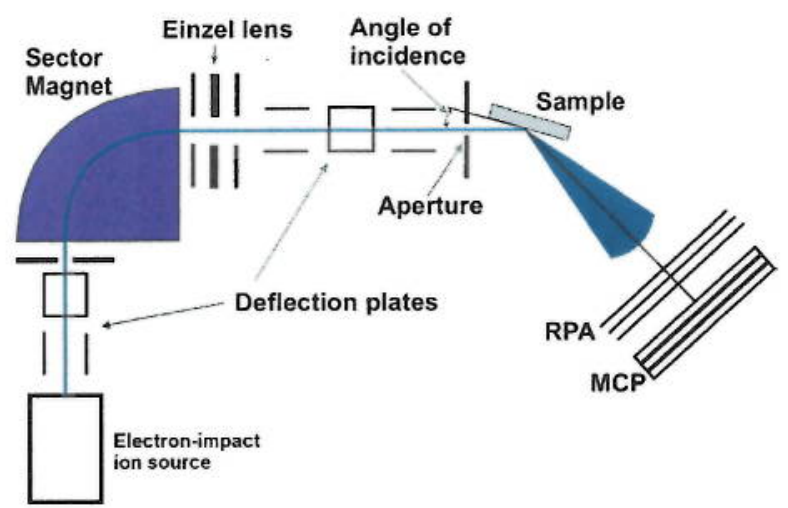

Fig. 1: Schematic measurement setup Key components of the ILENA test facility are an electron impact ion source, a sector magnet, a conversion surface, ion optics and an imaging micro channel plate detector (MCP) with a retarding potential analyser (RPA). For more details it is referred to the main text.

For our experiments, we used a beam of positively charged ions, although charge state conversion surfaces are needed for the conversion of neutrals, because ions can easily be guided and accelerated by electric and magnetic fields. However, positive ions are effectively neutralized prior to scattering off the conversion surface $[6,7]$, which justifies using positively charged ions for our experiments. Positive ions in the scattered particle beam are deflected by the positive potential of the RPA grid. Therefore only neutrals that are not ionized upon scattering and negative ions reach the detector. For the measurement of the negative ionization yield, the detector is floated on a negative potential so that only neutral atoms are detected. The portion of atoms that become negatively charged upon scattering off the test surface can be determined by the ratio of the detected particles with and without setting the detector to floating potential. A set of five measurements, three without and two with floating potential, is used to monitor time-dependent instabilities of the system [10].

To correct for the contribution by recoil sputtering, each measurement is repeated with noble gases since these do not form negative ions. The ionization yield measured for $\mathrm{He}$ and $\mathrm{Ne}$ is subtracted from the results of $\mathrm{H}$ and $\mathrm{O}$, respectively, as their masses are comparable.

The typical base pressure of the facility is about $5 \cdot 10^{-8}$ mbar that rises during operation of $(4.0 \pm 0.5) \cdot 10^{-7}$ mbar because of the introduction of the test gas. Prior to each set of measurements, the ion optics were optimized for maximum transmission throughout the experiment. The count rate was adjusted to a constant value to ensure the same statistics of about $6 \cdot 10^{5}$ counts in total for each measurement. 
The angular scattering was evaluated by analysis of the Full Width at Half Maximum (FWHM) of the angular scattering distribution. In the analyses the angular scattering in azimuthal and in polar direction were analyzed separately.

Except for energies below $500 \mathrm{eV}$, all measurements were performed with atomic ions. At energies below $500 \mathrm{eV}$, a reasonably high count rate could not be achieved with atomic ions and therefore, molecular ions were used for these measurements. Because about $80 \%$ of all molecules dissociate before reaching the conversion surface [6], under consideration of the atom energy, the analyses of these measurements are similar to the measurements conducted with atonic species. In these analyses it was assumed that the energy of a molecule is equally divided between the two atoms, which are formed by the dissociation. A relative error of $10 \%$ of the atom energy was assumed to consider cases deviating from the perfect bisection of the molecule energy.

\subsection{Boron-doped CVD Diamond}

Earlier experiments of scattering low energy neutral atoms on an undoped free-standing CVD diamond surface $((300 \pm 50) \mu \mathrm{m}$ thick) showed that the conductivity of the CS was not sufficient and electrical charging of the surface was observed [10]. This is not surprising as pure diamond is a very good insulator. Electrostatic charging of the sample during measurements resulted in deflection of the incoming ions, and thus in distorted angular scattering distributions, and generally in experiments not being able to be reproduced [10].

In [7], a natural type Ila diamond was investigated for stitability as a CS in an ENA detecting instrument, which did not charge during the scattering experiments. Natural diamond of this type contains only very few impurities, mainly $\mathrm{N}$ with a concentration of about $10^{18} \mathrm{~cm}^{-3}[15]$ but no $\mathrm{B}$ impurities [25]. The second type of natural diamond, type [15, is characterized by a higher electric conductivity than type Ila due to the abundance of B impurities of up to $10 \mathrm{ppm}$ (atomic fraction) [25]. The type lib diamond is a very rare material occurring in the natural world [15], but the synthetic equivalent is commercially available. Based on the physical parameters of conductivity and concentration of impurities of natural and synthetic diamond samples that were investigated in former studies [7,10], we concluded that electrostatic charging of a CVD sample used as a CS could be prevented by a B-concentration of about $10^{14} \mathrm{~cm}^{-3}$. According to the manufacturer [Diamond Materials GmbH, Hans-Bunte-Strasse 19, 79108 Freiburg i.B., Germanyl, the B-doped CVD sample, investigated in this study, contains $B$ with a concentration of $5 \cdot 10^{18} \mathrm{~cm}^{-3}$, which is equivalent to a Babundance of $28.4 \mathrm{ppm}$ (atomic fraction).

Similar to the experiments conducted in [10], we measured the variation of the angular scattering in long meastrement cycles for the B-doped CVD surface. In previous experiments it was observed that the mean angular scattering explicitly decreased over time due to electric fields caused by the built-up surface charge. These fields deflected the incoming ion beam and caused it to impinge at a different surface location on the CS. Any kind of significant change of the scattering angles in long measurement cycles under constant measurement conditions would indicate such electrostatic charging effects. 


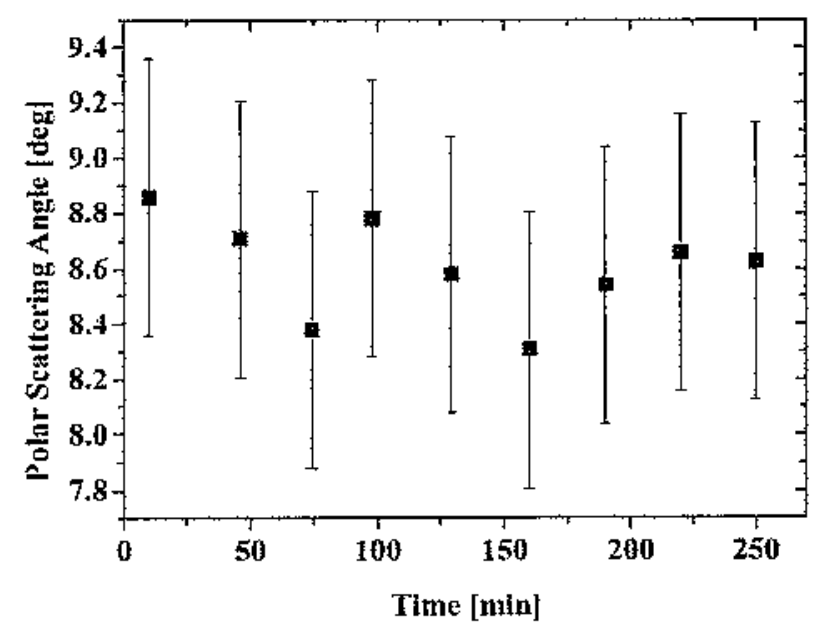

Fig. 2: Charging Experiment Scattering angle in polar direction for $(195 \div 19.5)$ e $\mathrm{H}_{2}$ impinging on the Buoped CID diamond as a function of time.

Figure 2 shows the mean angular scattering in polar direction as a function of time for nine measurements carried out with (195 \pm 19.5$) \mathrm{eV} \mathrm{H} \mathrm{H}_{2}$ under constant measurement conditions over the duration of 250 minutes. It shows that the results are reproduced within the measurenent accuracy of \pm 0.5 degree, thus surface charging is not observed. Additionally, also the angular scattering in azimuthal direction and the ionization yield were investigated for the measurements shown in Fig. 2. The results confirmed the reproducibility of the measurements, even with a measurement duration of 4 hours. It can be concluded that the B-atons in the diamond lattice of the sample lead to a sufficient increase of the electrical conductivity, hence preventing surface charging by the impinging ion beam.

For the chemical characterization of the B-doped CVD sample, especially for quantifying the abundance of $B$ in the sample, we measured the chemical composition of the sample with our Laser Ablation Ionization Mass Spectrometer (LIMS, instrument name LMS), which has been originally designed and built for space research at the University of Bern. A femtosecond laser system $(\lambda=775$ $\mathrm{nm}$, repetition rate of $\leq \mathrm{I} \mathrm{kHz}, \tau \sim 190 \mathrm{fs}$, energy/pulse $\leq 1 \mathrm{~mJ}$ ) is used for clean material ablation and ionization, coupled with a reflectron-type TOF mass analyzer. Details about LMS can be found in the numerous publications on the functionality and performance of the system, for example [16-20].

Figure 3 shows the mass spectrum of the B-doped CVD sample measured with LMS by applying a laser irradiance of about $12.5 \mathrm{TW} / \mathrm{cm}^{2}$. For the mass spectrum displayed in Fig. 3, spectra from 35'000 single laser shots were accumtlated in the histogram. The mass resolution of about $250 \pm 50$ is sufficient to distinguish between single masses and thus between isotopes. The relative abundance of ${ }^{12} \mathrm{C}$ and ${ }^{13} \mathrm{C}$ was measured to be $98.93 \%$ and $1.07 \%$ respectively, which is in good agreement with the terrestrial abundance of $98.89 \%$ and $1.11 \%$, respectively [21] and thus is a confirmation of good conditions for laser ablation measurements. 
a)
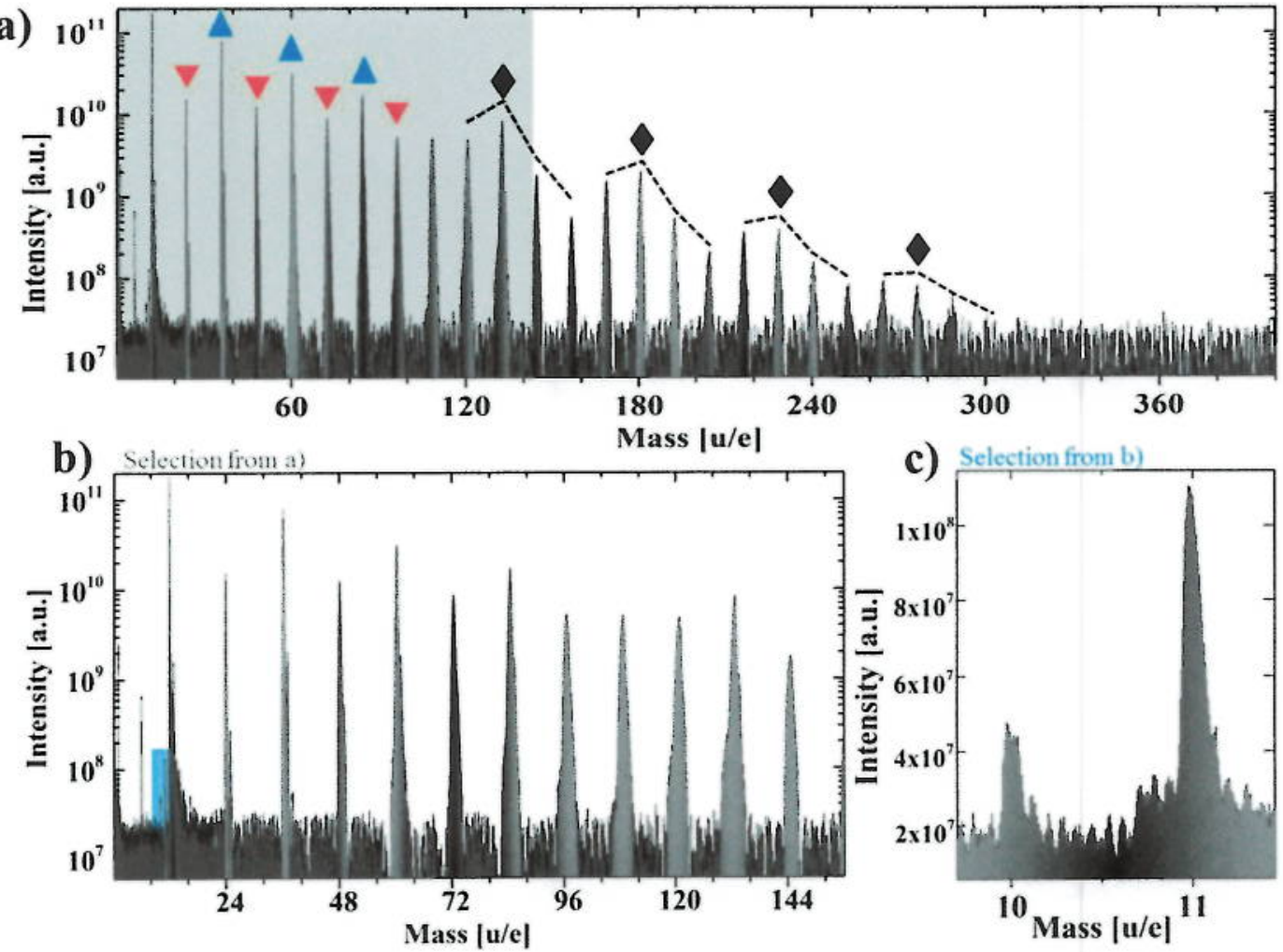

Fig. 3: Mass spectrum recorded with LMS showing the chemical composition of the B-doped CVD sample (a). Complete mass spectrum with odd (blue symbols) and even (red symbols) carbon cluster indicated, and black symbols show large carbon clusters with a periodicity of 4 atoms (b). Low-mass part of the mass spectrum showing the location of $B$ atoms; (c) zoom on the ${ }^{10} B$ and ${ }^{11} B$.

The chemical composition measurements showed the high purity of the synthetic diamond sample. From the production and handling of the sample, a possible abundance of $\mathrm{H}$ was expected, but only a very small signal of $\mathrm{H}^{+}$was visible in the mass spectrum. Additionally, we did not observe any $\mathrm{O}^{+}, \mathrm{N}^{+}$ or other possible contaminants in the mass spectrum. All mass peaks that were identified in the mass spectrum could be assigned to atomic $\mathrm{C}$, atomic $\mathrm{B}$ and $\mathrm{C}$ clusters. The carbon clusters formed in the plasma plume by recombination processes of the ablated C-atoms. C-clusters up to $\mathrm{C}_{24}$ are visible in the spectrum in Fig. 3a). We observed that clusters with an even number of C-atoms (marked by blue upwards pointing triangles in Fig. 3a) had higher signal intensities, thus were more likely to be formed than those with odd numbers of $\mathrm{C}$-atoms (red downwards pointing triangles in Fig. 3a). This is in agreement with the observations presented in [22], where the authors state the different processes and probabilities for $\mathrm{C}$-cluster ion formation induced by laser irradiation. Also in agreement with the research presented in [22], we observed that for clusters larger than $\mathrm{C}_{9}$ the odd and even rule change to a periodicity of 4 carbon atoms. In Figure 3 this is indicated by the black diamond shaped symbols and the dotted lines above the corresponding mass peaks, which were added to guide the eye.

For the quantitative analysis of the chemical composition of the B-doped CVD sample, all clusters were included in the calculations and the areas of the mass peaks were corrected by the according relative sensitivity coefficients (RSCs), which is necessary, as the sensitivity of the LMS is not the same in all species [20]. Based on the results of former studies [20,23], and based on the atomic orbital ionization potentials of the elements, the corresponding RSCs were applied, resulting in a composition of the sample of almost $100 \% \mathrm{C},(24.4 \pm 4.0) \mathrm{ppm}$ (atomic fraction) B and $(6.2 \pm 2.9) \mathrm{ppm}$ (atomic fraction) $\mathrm{H}$, which corresponds to the specifications given by the manufacturer.

The surface roughness is an important parameter affecting the angular scattering of the CS. Therefore, we conducted Atomic Force Microscope (AFM) measurements of the CVD sample to characterize the surface. Figure 4 shows the AFM image of the B-doped CVD diamond surface. The image covers an 
area of $20 \mu \mathrm{m} \times 20 \mu \mathrm{m}$, representing the typical sample surface, and a vertical scale of $8 \mathrm{~nm}$. AFM imaging was carried out in an ambient environment at room temperature employing Nanosurf FlexAFM scan head at the department of Chemistry and Biochemistry at the University of Bern. The average and root mean square surface roughness calculated from the whole image are $1.04 \mathrm{~nm}$ and $1.42 \mathrm{~nm}$, respectively.

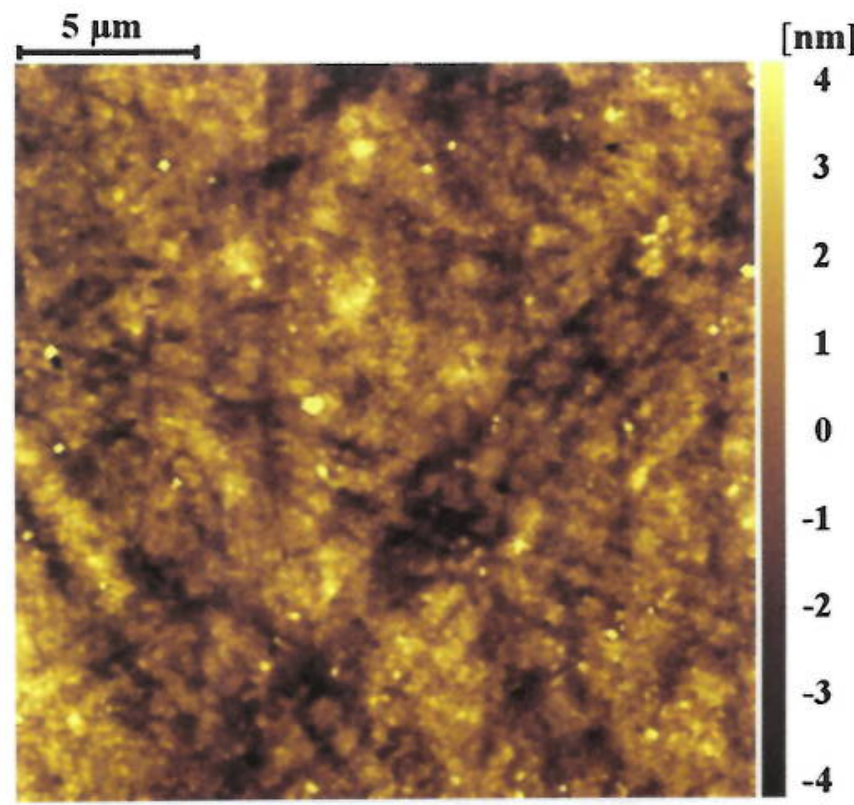

Fig. 4: AFM image

An area of $20 \mu \mathrm{m} \times 20 \mu \mathrm{m}$, representing the typical sample surface, was imaged with a Nanosurf FlexAFM scan head. The vertical extend of the surface roughness is $8 \mathrm{~nm}$ peak-to-valley.

\section{Results and Discussion}

\subsection{Angular Scattering}

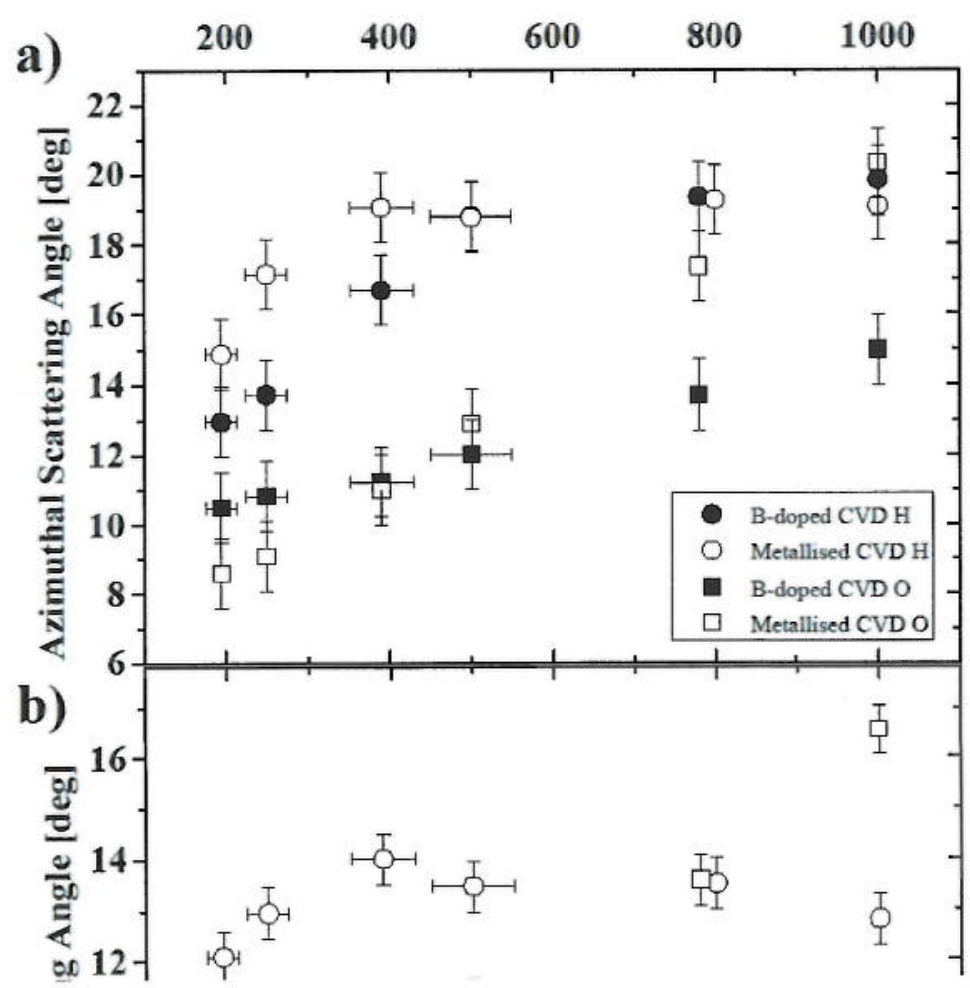

f the angular scattering distribution nents were carried out for $\mathrm{H}$ and $\mathrm{O}$ iple are compared to an undoped 
Fig. 5: Angular scattering

The angular scattering in

azimuthal (a) and polar (b)

direction was meastured for the

$B$-doped and the metallised

[10] CID diamond at an

angle of incidence of $8^{\circ}$.

Measturements show' with an

energ' uncertainty' were

carried oul with molecules.

CVD diamond with a metallized backside, presented in [10]. Figure 5a)

scattering in azimuthal and polar direction, respectively. The energy of the atoms is considered with a relative error of $10 \%$ for the measurements that were carried out using $\mathrm{H}_{2}$ and $\mathrm{O}_{2}$, for the reasons mentioned in Sec. 2.1. An increase of the angular scattering, in both azimuthal and polar direction, is observed with increasing incident energies, which is in agreement with the findings from earlier publications $[8,10,14]$.

For the B-doped CVD diamond sample, the measurements of the angular scattering in azimuthat direction resulted in $13^{\circ}$ to $20^{\circ}$ (FWHM) for measurements with $\mathrm{H}$ and $10^{\circ}$ to $15^{\circ}$ (FWHM) for O. For $\mathrm{H}$ at energies below $500 \mathrm{eV}$ this corresponds to a narrower angular scattering than reported for the metallized CVD diamond [10], while the scattering distributions are comparable to the sample investigated in [10] with energies of $500 \mathrm{eV}$ and above (Fig. 5a). For measurements with $\mathrm{O}$ and energies below $390 \mathrm{eV}$, the angular scattering in azimuthal direction is slightly higher for the B-doped CVD diamond than the results reported in [10]. On the other hand the angular scattering of $O$ in azimuthal direction is significantly snaller for the B-CVD sample for energies between $780 \mathrm{eV}$ and $1000 \mathrm{eV}$, compared to the metallized CVD sample.

Figure $5 \mathrm{~b}$ ) shows the angular scattering in polar direction measured for $\mathrm{H}$ and $\mathrm{O}$ on the $\mathrm{B}$-doped $\mathrm{CVD}$ sample, and the data measured on the metallized CVD sample [10] for comparison. We measured a scattering in polar direction of $8.5^{\circ}$ to $11^{\circ}$ for $\mathrm{H}$ and $7.5^{\circ}$ to $10.5^{\circ}$ for $\mathrm{O}$. Compared to the metallized CVD diamond, the scattering properties of the B-doped CVD diamond are significantly better in polar direction. Only for $\mathrm{O}$ at $195 \mathrm{eV}$ are the angles comparable. For all other evaluated measurements significantly narrower angular scattering in polar direction is found for the B-doped CVD sample.

\subsection{Negative Ionization Yield}

The negative ionization yieid is of major interest for CSs in space plasma research [23] as it contributes directly to the total detection efficiency of an ENA detecting instrument. The negative ionization yield is defined as the quotient of the registered number of negative ions to the number of neutrals.

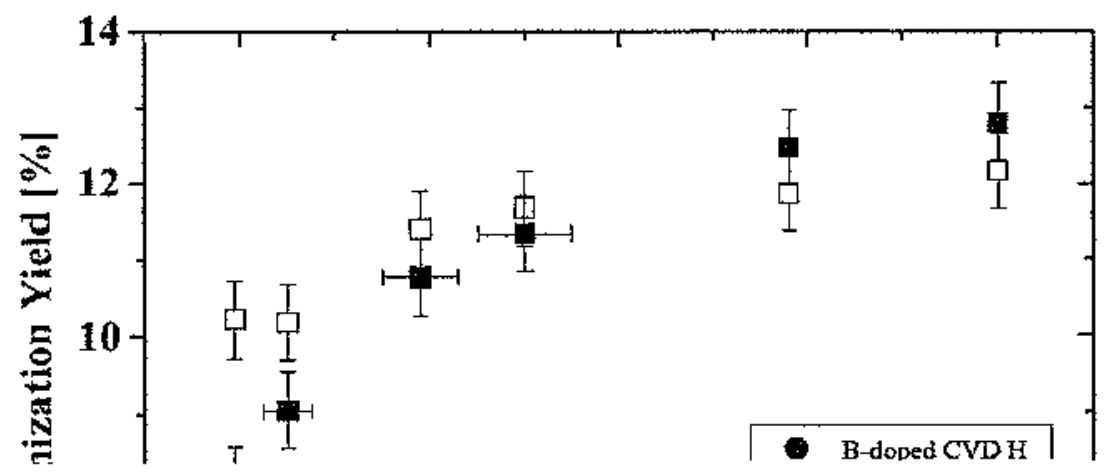


Fig. 6: Negative ionization yield

Meastrements of the ionisation yield for $H$ and $O$, carried out at an angle of incidence of $8^{\circ}$. Results of the metallised CVD diamond from [10] are shown for comparison. Meastrements shown with an energy uncertainty were carried out with molecules.

Measurements were carried out at an angle of incidence of $8^{\circ}$ for $\mathrm{H}$ and $\mathrm{O}$. The data were corrected for the recoil sputtering background using the measurements of He and Ne, respectively. In Figure 6 the results are shown together with the data of the metallized CVD diamond from [10]. For the B-doped CVD diamond, we measured a negative ionization yield between 8 and $13 \%$, depending on the atom energy, for $\mathrm{O}$. The negative ionization yield of $\mathrm{H}$ was measured to be about $2 \%$, independent of the atom energy.

The measured negative ionization yields for $\mathrm{H}$ are similar to the previous measurements with the metallized CVD diamond sample [10] within the range of uncertainties. For $\mathrm{O}$ the $\mathrm{B}$-doped CVD diamond sample shows a stronger energy dependency than the metallized CVD sample. In comparison to the metallized CVD sample the negative ionizations yield measured for the B-doped sample is lower for energies below, but higher for energies above $500 \mathrm{eV}$.

\subsection{Figure of Merit}

A. high negative ionization yield and narrow angular scattering distribution are preferred for the application of a CS in an ENA imaging instrument. In [10] a Figure of Merit (FoM) was introduced to combine these two key variables. There the FoM is defined as the negative ionization yield divided by the fraction of the hemisphere above the CS that is covered by the scattered atoms. The resulting FoM is unitless and should preferably be large quantity [10].

In Figure 7a) the FoM resulting from the measured angular scattering and ionization yield (Fig. 5, Fig. 6) is shown as a function of atom energy for an angle of incidence of $8^{\circ}$. For comparison the data for the metallized CVD diamond [10] were added to the graph. Compared to the metallized CVD diamond, the B-doped CVD diamond sample shows a slightly better performance for $\mathrm{H}$ over the whole range of energies covered by our experiments. For $O$ the FoM decreases with increasing energy for the metallized CVD diamond due to the steep increase of the angular scattering with increasing atom energy (Fig. 9 in [10]). For the B-doped CVD diamond sample we find a FoM that is constant within the range of uncertainties over the investigated range of energies. Thus, for the measurement with $\mathrm{O}$ 
the metallized CVD diamond sample shows a better performance for energies $<250 \mathrm{eV}$, while the Bdoped CVD diamond sample is favorable for energies $>390 \mathrm{eV}$.

In Figure $7 \mathrm{~b}$ ) the FoM of $\mathrm{O}$ for different angles of incidence is displayed as a function of the incident atom energy. Data points of measurements at a constant angle of incidence are connected by a line to guide the eye. For comparison, the data of the metallized CVD diamond sample from [10] are shown, too. It can be observed that the B-doped CVD diamond sample performs significantly better than the metallized CVD diamond at small angles of incidence and particularly at high atom energies. For larger angles of incidence, the difference in the FoM can still be observed, but becomes less important.

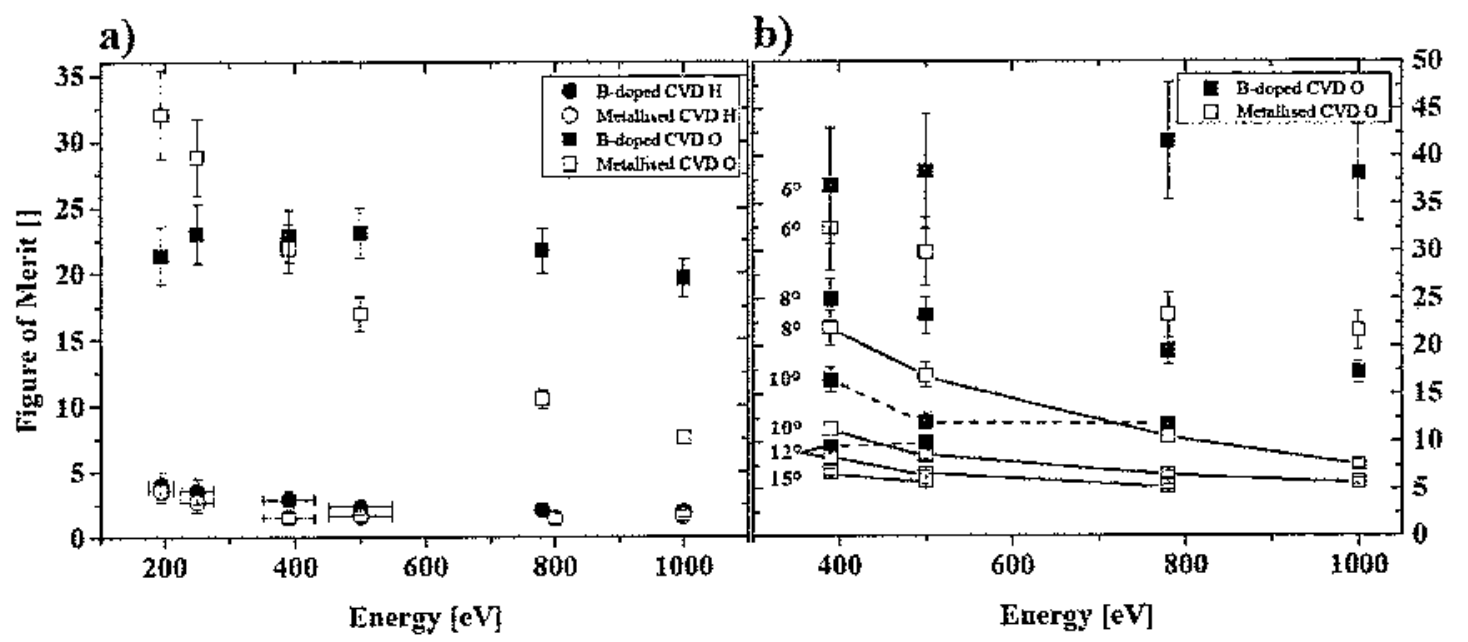

Fig. 7: Figure of Merit

a) The Figure of Merit in dependence of atom energy for $H$ and $O$ at an angle of incidence of $8^{\circ}$ on the B-doped CVD compared to the values measured on a CVD sample with a metallic coating [10].

b) Figure of Merit for different angles of incidence for O on both CVD diamond samples. For reasons of clarity, data points measured at larger angles of incidence are connected by lines.

The FoM is defined here very general since the evaluation of the CS is not done for a specific ENA instrument with a known angular acceptance in polar and azimuth direction. However, the choice of FoM definition is not that critical for the qualitative assessment of a CS. For example, if we use the scattering in the azimuth angle instead of the fraction of the hemisphere in the FoM we arrive at the same conclusion of the B-doped CVD diamond sample being superior to the metallized CVD diamond.

\section{Conclusion}

A B-doped CVD diamond from Diamond Materials GmbH was tested in the ILENA test facility at the University of Bern [13] for suitability as a conversion surface in an ENA imaging instrument for future space missions, such as NASA's Interstellar Mapping Probe (IMAP). The B-dotation was chosen to prevent possible electrostatic charging of the conversion surface upon ionization of impinging neutral atoms. Such charging effects were observed in earlier measurements of a pure CVD diamond sample [10].

For characterization of the B-doped CVD diamond sample, we measured the B-abundance in the sample with our LIMS instrument [16-20]. Our measurements result in an abundance of $(24.4 \pm 4.0) \mathrm{ppm} \mathrm{B}$ (atomic fraction) in a pure C-matrix, which is in agreement with the specifications given by the manufacturer. Despite the high sensitivity of the LMS we did not observe any contaminants in the sample (Fig. 3), which could possibly occur due to manufacture processes and 
handling of the sample. During the subsequent ILENA measurements electrostatic charging of the sample was not observed, even during long duration measurements up to 250 minutes. This is in agreement with our estimations of the dopant concentration needed for preventing electrostatic charging, performed prior to the experiments.

The surface roughness of the B-doped sample was determined by AFM imaging. With a restit of Ra $\approx 1.0 \mathrm{~nm}$ ( $8 \mathrm{~nm}$ peak to valley) the sample seems to have an equally smooth surface as the metallized and the pure CVD diamond sample investigated in [10], where $R a \approx 1.3 \mathrm{~nm}$ was reported, despite that the B implantation in the diamond may lead to a higher surface roughness. Furthermore, the AFM imaging was carried out after the measurements in the ILENA facility and after chemical composition measurements with our LIMS instrument.

The angular scattering in both polar and azimuthal directions and the negative ionization yield were measured in the ILENA test facility for $\mathrm{H}$ and $\mathrm{O}$ covering an energy range between of $195 \mathrm{eV}$ and $1 \mathrm{keV}$. In comparison to a metallized CVD dianond [10], we measured smaller angular scattering in azimuthal direction for $\mathrm{H}$ for energies below $500 \mathrm{eV}$ and comparable values for energies above (Fig. 5a). However, the angular scattering in azimuthal direction for $\mathrm{H}$ as a function of energy shows a smoother increase for the B-doped sample than for the metallized CVD diamond sample. For $\mathrm{O}$, in comparison to the metallized sample, we measured a larger angular scattering in azimuthal direction for low energies, but smaller angles for higher energies, which results in an even smoother increase of the angular scattering with energy (Fig. 5a). In general, small angular scatter and a reduced dependence of the angular scattering on atom energy are of high advantage for the ion-optical design of an ENA imaging instrument and for its calibration. The smaller angular scattering means less particle loss and allows for highly optimized ion optics and thus, for a higher angular resolution of the instrument.

For the angular scattering in polar direction, we measured clearly smaller, thus favorable angular scatter than it was reported for the metalized CVD diamond sample. The angular scattering in polar direction is mainly influenced by the scattering geometry, thus by the angle of incidence. Similarly to the angular scattering in azimuthal direction, the polar scattering affects the design of an ENA imaging instrument. A smaller polar angle of the scattered ionized neutral atoms allows for a more compact design of the instrument, higher optimization of ion optics and therefore, for higher angular resolution of the measurements in space. From these results we conclude that the properties of the B-doped CVD diamond $\mathrm{CS}$ are advantageous for its application in an ENA imager for space research.

From our measurements in the ILENA test facility, we determined the negative ionization yield of the B-doped CVD diamond sample. For H incident on the B-doped CVD diamond surface in an angle of $8^{\circ}$, we measured an ionization yield of about $2 \%$, which is a value comparable to the ionization yield determined for the metallized CVD diamond sample (Fig. 6). For $\mathrm{O}$ at an angle of incidence of $8^{\circ}$ on the B-doped sample, we determined, in comparison to the metallized sample, a lower negative ionization yields for energies below $500 \mathrm{eV}$ and higher for measurements above $500 \mathrm{eV}$. Since the negative ionization yield reaches a saturation value at high energies, this saturation level of about $13 \%$ is slightly higher for the B-doped CVD diamond sample than for the metallized sample, which reaches an ionization yield of about $12 \%$ at energies above $800 \mathrm{eV}$.

For the investigation, how the angular scattering and the negative ionization yield can counterbalance each other, the Figure of Merit (FoM) was used. For H, the FoM derived from the measurements on the B-doped sample for $8^{\circ}$ incidence are comparable to the metallized CVD diamond sample. For $O$ we find, in comparison to the metallized CVD diamond sample, a higher FoM for energies above $390 \mathrm{eV}$ (Fig, 7a). In addition, measurements were carried out for angles of incidence of $6^{\circ}, 10^{\circ}, 12^{\circ}$ and $15^{\circ}$ (Fig. 7 b). Similar to the results reported in [10], we observe a clear increase of the FoM with decreasing angle of incidence. For smaller angles, $6^{\circ}$ for example, the much narrower angular scattering distribution clearly compensates for the smaller negative ionization yield, which results from the measurements at these smalt angles of incidence. Accordingly the much larger angular scatter 
for larger angles of incidence camnot be compensated by the slightly higher negative ionization yield. These results show that although the ionization yield is one of the main constraints of the detection efficiency of an ENA imaging instrument, the angular scattering is of great importance, too. The scattering properties of a CS are not only important for the optimization of the instrument design, but our analyses show that small angular scattering can outbalance the ionization yield.

In [10] it was shown that highly polished CVD diamond is a material with promising properties compared to alternatives, like for example $\mathrm{Al}_{2} \mathrm{O}_{3}[14]$ or to the H-terninated diamond like carbon CSs, which were applied for example in the IBEX-Lo camera [8]. The chemical inertness and especially the off-the-shelf availability of the samples emplasize the advantages of CVD diamond for its use as a CS in ENA imaging space instrumentation.

The FoM shows that the performance of the B-doped CVD diamond sample outperforms the metallized sample, which is the most distinct for small angles of incidence. This better performance can be ascribed to the smailer angtlar scattering, which is, among others, an impotant driver of the total transmission and thus detection efficiency of an ENA space instrument.

\section{Acknowledgment}

This work is supported by the Swiss National Science Foundation. We would like to thank llya Pobelov from the department of Chemistry and Biochemistry at the University of Bern for the AFM measurements. We also thank Dr. Eckhard Worner, CEO of Diamond Materiats GmbH, for his consulting services regarding CVD diamond. 


\section{References}

[1] P. Wurz, Detection of Energetic Neutral Particles, The Outer Heliosphere: Beyond the Planets, (eds. K. Scherer, H. Fichtner, E. Marsch), Copernicus Gesellschaft e.V., Katlenburg-Lindau, Germany (2000) 251-288.

[2] Y. Kazana, S. Barabash, M. Wieser, K. Asamura, P. Wurz, Development of an LENA instrument for planetary missions by numerical simulations, Planet. Space Sci. 55 (2007) 1518-1529.

[3] S.A. Fuselier, A.G. Ghielmetti, E. Hertzberg, A.S. Moore, D. Isaac, J.W. Hamilton, C. Tillier, E. Möbius, M.S. Granoff, D. Heirtzler, B. King, H. Kucharek, S. Longworth, J. Nolin, S. Turco, S. Ellis, J. Googins, F. Kudirka, J. Tyler, M. Vosbury, G. Clark, M. O'Neal, P. Wurz, J.A. Scheer, L.A. Saul, D. Piazza, P. Bochsler, M. Wieser, C. Schlemm, D.1. McComas, J. Scherrer, S. Pope, H.O. Funsten, D. Chornay, J. Lobell, T.E. Moore, P. Rosmarynowski, T. Friedmann, R.J. Nemanich, The IBEX-Lo Sensor, Space Science Review 146 (2009) 117-147.

[4] A. Vorburger, P. Wurz, S. Barabash, M. Wieser, Y. Futaana, C. Lue, M. Holmström, A. Bhardwaj, M.B. Dhanya, K. Asamura, Energetic Neutral Atom lmaging of the Lunar Surface, Jou. Geophys. Res. $118(7)$ (2013) 3937-3945.

[5] A. Galti, P. Wurz, S.A. Fuselier, D.J. McComas, M. Bzowski, J.M. Sokół, M.A. Kubiak, E. Möbius, "lmaging the heliosphere using neutral atoms from solar wind energy down to 15 eV," Astrophys. Jou. 796 (2014) 9 (18pp).

[6] P. Wurz, R. Schletti, M. Aeltig, Hydrogen and oxygen negative ion production by surface ionization using diamond surfaces, Surf. Sci. 373 (1997) 56-66.

[7] J.A. Scheer, K. Brüning, T. Fröhlich, P. Wurz, W. Heiland, Scattering of small molecules from a diamond surface, Nucl. Instrum. Methods B157 (1999) 208-213.

[8] P. Wahlström, J. Scheer, P. Wurz, E. Hertzberg, S. Fuselier, Calibration of Charge State Conversion Surfaces for Neutral Particle Detectors, Jou. Appl. Phys. 104 (2008) 034503-1 034503-6, DOF: $10.1063 / 1.2957064$.

[9] J. Lienemann, D. Blauth, S. Wethekam, M. Busch, H. Winter, P. Wurz, S.A. Fuselier, and E. Hertzberg, Negative ion formation during scattering of fast ions from diamond-like carbon surfaces, Nucl. Instr. Meth. B269 (2011) 915-918.

[10] M.B. Neuland, A. Riedo. J.A. Scheer, P. Wurz, Self-supporting CVD diamond charge state conversion surfaces for high resolution imaging of low-energy neutral atoms in space plasmas, Appl. Surf. Sci. 313 (2014) 293-303.

[11] N. A. Schwadron, M. Opher, J. Kasper, R. Mewaldt, E. Moebius, H. E. Spence, T.H. Zurbuchen, lnterstellar mapping and Acceleration Probe (IMAP), J. Phys.: Conference Ser. 767 (2016) 012025 .

[12] M. Wieser. Y. Futaana, S. Barabash, P. Wurz, Emission of energetic neutral atoms from water ice under Ganymede surface-like conditions, lcarus 269 (2016) 91-97.

[13] P. Wahlström, J.A. Scheer, A. Riedo, P. Wurz, Test Facility to Study Surface-Interaction Processes for Particle Detection in Space, J. Spacecr. Rocket 50 (2) (2013) 402-410. 
[14] A. Riedo, M. Ruosch, M. Frenz, J.A. Scheer, P. Wurz, On the surface characterization of an $\mathrm{Al}_{2} \mathrm{O}_{3}$ charge state conversion surface using ion scattering and atomic force microscope measurements, Appl. Surf. Sci. 258 (2012) $7292-7298$.

[15] M.A. Prelas, G. Popovici, L.K. Bigelow, ed., Handbook of industrial diamonds and diamond films, Marcel Dekker Inc, New York, 1998.

[16] U. Rohner, J. Whitby, P. Wurz, A miniature laser ablation time-of-flight mass spectrometer for planetary rover, Rev. Sci. Instrum. 75(5) (2003) 1314-1322.

[17] V. Grimaudo, P. Moreno-Garcia, A. Riedo, S. Meyer, M. Tulej, M.B. Neuland, M. Mohos, C. Gütz, S.R. Waldvogel, P. Wurz, P. Broekmann, Toward Three-Dimensional Chemical lmaging of Ternary $\mathrm{Cu}-\mathrm{Sn}-\mathrm{Pb}$ Alloys Using Femtosecond Laser Ablation/lonization Mass Spectrometry, Anal. Chem., 89 (2017) 1632 - 1641.

[18] A. Riedo, A. Bieler, M.B. Neuland, M. Tulej, P. Wurz, Performance evaluation of a miniattre laser ablation time-of-flight mass spectrometer designed for in situ investigations in planetary space research, J. Mass Sepctron. 48 (2013) 1 - 15.

[19] A. Riedo, M.B. Neuland, S. Meyer, M. Tulej, P. Wurz, Coupling of LMS with a fs-laser ablation ion source: elemental and isotope composition measurements. JAAS 28(8) (2013) 1133-1356.

[20] M.B. Neuland, V. Grimaudo, K. Mezger, P. Moreno-García, A. Riedo, M. Tulej, P. Wurz, Quantitative measurement of the chemical composition of geological standards with a miniature laser ablation/ionization mass spectrometer designed for in situ application in space research, Meas. Sci. Technol. 27 (2016) 035904 (13pp).

[2i] J. Meija, T.B. Coplen, M. Berglund, W.A. Brand, P. De Bièvre, M. Gröning, N.E. Holden, J. Irrgeher, R.D. Loss, T. Walczyk, T. Prohaska, Isotopic compositions of the elements 2013 (IUPAC Technical Report), Pure Appl. Chem. 88(3) (2016) 293-306.

[22] N. Fürstenat, F. Hillenkamp, R. Nitsche, Laser-induced positive and negative molecular ions from thin carbon foils, Int. J. Mass Spectrom. Ion Phys. 31 (1979) 85-91.

[23] P. Wurz, J. Scheer, M. Wieser, Particte Scattering of Surfaces: Appication in Space Science, Surf. Sci. Nanotech. Vol. 4 (2006) 394-400.

[24] M. Wieser, P. Wurz, E. Moebius, The ion-optical protoype of the low energy neutral atom sensor of the Interstellar Boundary Explorer Mission (IBEX), Scientific Instruments 78(12) (2008) 124502 .

[25] Ch.M. Breeding and J.E. Shigley, The "type" classification system of diamonds and its importance in gemology, Gems Gemology, Vol. 45(2) (2009) 96-111. 